\title{
Um ensaio sobre a experiência educacional na programação de computadores: a abordagem tradicional versus a aprendizagem baseada em projetos
}

\author{
Alexandre Grotta ${ }^{1}$, Edmir P. V. Prado ${ }^{1}$ \\ ${ }^{1}$ Programa de Pós-graduação em Sistemas de Informação - Universidade de São Paulo \\ Rua Arlindo Béttio, 1000, São Paulo - SP, 03828-000 \\ grotta@ifsp.edu.br, eprado@usp.br
}

\begin{abstract}
Towards the innovation on computer programming education, there are reports about changes of two educational aspects. First, the use of constructivist teaching methods like project-based learning (PjBL). Second, the measuring non-mandatory metrics like motivation. But there are also reports about the challenges on measuring and evaluating these changes. Thus, given the educational continuum on computer programming education, what and how to evaluate, regarding the teaching and learning via PjBL versus the traditional educational experience? Based on the literature review, this essay proposes a common pattern to evaluate them. Regarding the teaching, we propose to evaluate the teaching methods and the educational resources. Regarding learning results, we propose to evaluate supportive metrics, like the motivation to learn and the cognitive gap. As future discussions, this pattern might be extended to related computational education and future research.
\end{abstract}

Resumo. Para inovar na educação em programação de computadores, há relatos de mudanças em dois aspectos educacionais. Primeiro, uma maior utilização de métodos construtivistas de ensino, tais como a aprendizagem baseada em projetos (PjBL). Segundo, a introdução de avaliações auxiliares, tais como a motivação dos alunos. No entanto, há também relatos de desafios para a avaliação destes dois aspectos. Assim, dado o continuum educacional de programação de computadores, o que avaliar, e como avaliar, no ensino PjBL, com relação à experiência educacional tradicional ? Embasado em uma revisão de literatura, este ensaio propõem um padrão para estas avaliações, a partir da visão do ensino e da aprendizagem. Primeiro, com relação ao ensino, avaliar métodos e recursos educacionais. Segundo, com relação à aprendizagem, utilizar avaliações auxiliares, tais como a motivação para aprender ou a lacuna cognitiva. Por fim é discutida a possibilidade de que este padrão venha a ser estendido ao ensino computacional e a pesquisas futuras.

\section{Introdução}

Elevadas taxas de evasão têm atingido o ensino computacional de nível superior, tais como cursos de Ciência da Computação e de Sistemas de Informação, inclusive no contexto de países desenvolvidos. A educação computacional é desafiante. A aprendizagem nesta área do conhecimento usualmente requer maior capacidade técnica dos alunos. Neste contexto, o ensino e aprendizagem de programação de computadores (EA-PROG) é uma área fundamental e também desafiadora. O EA-PROG é complexo e laborioso. Demanda alta 
carga cognitiva dos alunos e possui longa curva de aprendizagem. Devido a estas características, há relatos de um alto nível de evasão dos alunos em cursos introdutórios. Além destes desafios, as novas gerações de alunos irão programar smartphones, robôs, entre outras máquinas computacionais cada vez mais presentes na sociedade, em complemento aos computadores como são atualmente conhecidos [Chan Mow 2008; Giraffa et al. 2014; Iskander 2008; Queirós 2014; Silva Filho et al. 2007].

Diante deste cenário, há dois relevantes aspectos para pesquisas educacionais no EA-PROG. Primeiro, há um forte debate na educação a respeito dos méritos da educação construtivista versus a educação instruída. Segundo, há relatos da utilização de avaliações auxiliares no EA-PROG, tais como a motivação dos alunos [Queirós 2014; Boruchovitch 2008].

A abordagem construtivista, também referenciada como centrada no aluno, tende a diminuir a lacuna cognitiva existente entre diferentes alunos, pois utiliza o nível cognitivo mais espontâneo e próximo aos estudantes. De fato, a educação pode ser definida a partir de polos bastantes distintos, até mesmo opostos entre si: o ensino instrutivista e o ensino construtivista. Estes polos do ensino são denominados visões ou abordagens. Cada uma dessas duas visões é aplicada na educação por meio de métodos específicos de ensino, no qual a Aprendizagem Baseada em Projetos (PjBL) é um representante da visão construtivista. Por outro lado, além das inovações nos métodos de EA-PROG, há também relatos de pesquisas que utilizaram avaliações auxiliares, tais como a motivação para aprender e o engajamento dos alunos, em complemento às avaliações educacionais tradicionais, tais como as notas [Biggs and Tang 2011; Boruchovitch 2008; Fior and Mercuri 2013; Goulding 2013; Guzman-Ramirez and Garcia 2013; Manogaran 2013; Payne 2009].

Com base em uma revisão de literatura, este ensaio propõe um padrão para as avaliações no EA-PROG. Primeiro, é proposto avaliar o ensino por meio da experiência educacional, seus métodos e recursos educacionais. Para avaliar os resultados estudantis, é proposto a utilização de avaliações auxiliares, tais como a motivação, o engajamento ou a lacuna cognitiva dos alunos. Em complemento a esta seção, o ensaio possui outras seis seções. A Seção 2 apresenta a fundamentação teórica. A Seção 3 apresenta o objetivo do ensaio, seguida pela proposta do ensaio na Seção 4. As limitações e discussões estão disponíveis na Seção 5. Na Seção 6 são tecidas as conclusões.

\section{Fundamentação Teórica}

Esta seção apresenta os conceitos fundamentais utilizados por este ensaio. No EA-PROG é plausível considerar o instrutivismo e o construtivismo como duas metáforas que ajudam a compor a visão do todo, ocupando os extremos do continuum educacional. Para compreender o todo, se faz necessário primeiramente entender estes dois polos [Payne 2009; Porcaro 2011]. Assim, a subseção 2.1 dispõe uma breve introdução às visões instrutivista e construtivista no EA-PROG. A subseção 2.2 detalha PjBL como método

educacional construtivista no EA-PROG. Por fim, a subseção 2.3 fundamenta os fatores tradicionais de avaliação da aprendizagem.

\subsection{Ensino Instrutivista de Programação de Computadores}

A visão instrutivista é assim denominada pois o ensino ocorre de modo instruído. Há um fluxo de aprendizagem do educador para os educandos, instruindo-lhes novos 
conhecimentos. Também é referenciada como abordagem centrada no professor, bem como abordagem tradicional ou clássica, pois é a predominante no ambiente educacional. A abordagem instrutivista também pode ser referenciada como passiva, uma vez que o aluno recebe a instrução [Biggs and Tang 2011; Payne 2009].

De modo similar ao que ocorre na educação em geral, a literatura aponta que o EA-PROG é influenciado pelas visões instrutivista e construtivista de ensino. A visão tradicional do EA-PROG continua sendo a instrutivista. As aulas consideradas tradicionais em EA-PROG são instrutivistas: expositivas, usualmente seguidas por provas de validação de entendimento dos conceitos por meio de questões. O EA-PROG tradicional é voltado para atividades de escrever códigos e aprender a sintaxe da linguagem de programação. Tende a ser verticalizado, com foco em determinada disciplina ou tecnologia, sem integrar diversos tópicos ou mesmo integrar disciplinas entre si [Corno and De Russis 2017; Goulding 2013; Greening 2012].

Por outro lado, o construtivismo tem sido uma das filosofias de maior influência na educação recente. A abordagem construtivista é referenciada como aprendizagem ativa, aprendizagem construída ou centrada no aluno, pois a educação se constrói a partir do aluno. No EA-PROG, o ensino construtivista se apresenta como alternativa ao ensino tradicional. Estudos apontam diversas vantagens da abordagem construtivista sobre a abordagem instrutivista para a educação na área de tecnologia. Exemplos de métodos de ensino construtivistas são: discussões iterativas, aprendizagem coletiva, aprendizagem baseada em problema (PBL), aprender pelo fazer (do inglês learn by doing, tradução nossa), com especial relevância para PjBL [Beck and Kosnik 2012; Biggs and Tang 2011; Greening 2012; Guzman-Ramirez and Garcia 2013; Payne 2009; Zhang and Liu 2012].

\subsection{O que é Aprendizagem Baseada em Projetos}

PjBL pode ser definida como a teoria e a prática da utilização de projetos próximos à vida real dos estudantes. Tem como objetivo facilitar a aprendizagem, individual e coletiva, maximizando-se a performance em um ambiente de aprendizagem por meio do fazer. Há inclusive uma forte concordância sobre os grandes potenciais educacionais da PjBL para a educação tecnológica. PjBL é baseada no conceito construtivista de aprendizagem. PjBL também pode ser definida como uma abordagem de ensino e aprendizagem que se propõe a engajar alunos em um contexto autêntico, exploratório e orientado à resolução de problemas [DeFillippi 2001; Guzman-Ramirez and Garcia 2013; Mioduser and Betzer 2007; Romeike and Göttel 2012]. Neste sentido, projetos e produtos finais guiam a construção do conhecimento, pautados em contextos próximos à realidade dos alunos, de modo colaborativo, envolvendo-os em investigações e atividades de design, como apoio de tecnologias de aprendizagem e recursos educacionais voltados a PjBL.

\subsection{Fatores para avaliação de aprendizagem em programação de computadores}

Esta subseção fundamenta dois fatores para avaliação da aprendizagem. Primeiro, a avaliação do rendimento escolar e sua relação com o ensino tradicional. Segundo, avaliações auxiliarias, tais como a motivação para aprender dos alunos. O rendimento escolar, por vezes representado pelas notas, é uma avaliação fundamental dentro do ambiente educacional. Atrelado ao rendimento escolar, é necessário avaliar a presença dos alunos no contexto do ensino presencial [Luckesi 2008]. No Brasil, esta prática é regulamentada pela Lei de Diretrizes e Bases da Educação Nacional, cujo objetivo final é verificação do acúmulo de conhecimento adquirido, bem como a avaliação da 
frequência mínima requerida por Lei [BRASIL 1996]. Portanto, o rendimento escolar e a frequência escolar são avaliações indispensáveis no contexto do ensino superior presencial de computação.

Por outro lado, avaliações auxiliares têm sido relevantes no contexto internacional na educação em vários segmentos de escolarização. A motivação para aprender (doravante denominada motivação) é uma das principais avaliações auxiliares, portanto abordada por este ensaio. A motivação possui características preditivas de eventos no contexto escolar [Boruchovitch 2008]. Já o engajamento pode ser definido como "a relação que se estabelece entre o aluno e uma atividade escolar" (STELKO-PEREIRA; VALLE; WILLIAMS, 2015, pág. 207). Além disso, é aceita a correlação entre motivação, engajamento escolar e interesse escolar [Fior and Mercuri 2013].

\section{Objetivo}

Com base no contexto apresentado, o objetivo deste ensaio é propor um padrão para o que avaliar, e como avaliar, no EA-PROG por meio de PjBL, quando comparado ao EAPROG tradicional. Para tornar a proposta mais compreensível, parte-se de um exemplo teórico e dois perfis de alunos T e F, todos obtidos da obra de Biggs e Tang (2011) e adaptados ao contexto de uma disciplina de ensino de programação. O primeiro perfil, nomeado $\mathrm{F}$, possui menor afinidade acadêmica e um histórico de pior rendimento escolar. Nas aulas tradicionais, o perfil $\mathrm{F}$ não faz muitas perguntas pois seu conhecimento prévio é mais limitado: seu objetivo é basicamente ser aprovado. O segundo perfil, nomeado T, é mais acostumado ao meio acadêmico e possui um bom histórico de rendimento. Utiliza seu conhecimento prévio para formular questões e acompanhar o que está sendo ensinado nesta aula tradicional. $\mathrm{O}$ perfil $\mathrm{T}$ parece mais engajado às aulas tradicionais de EA-PROG do que o perfil $\mathrm{F}$, conforme observado no lado direito da figura 1 a seguir:

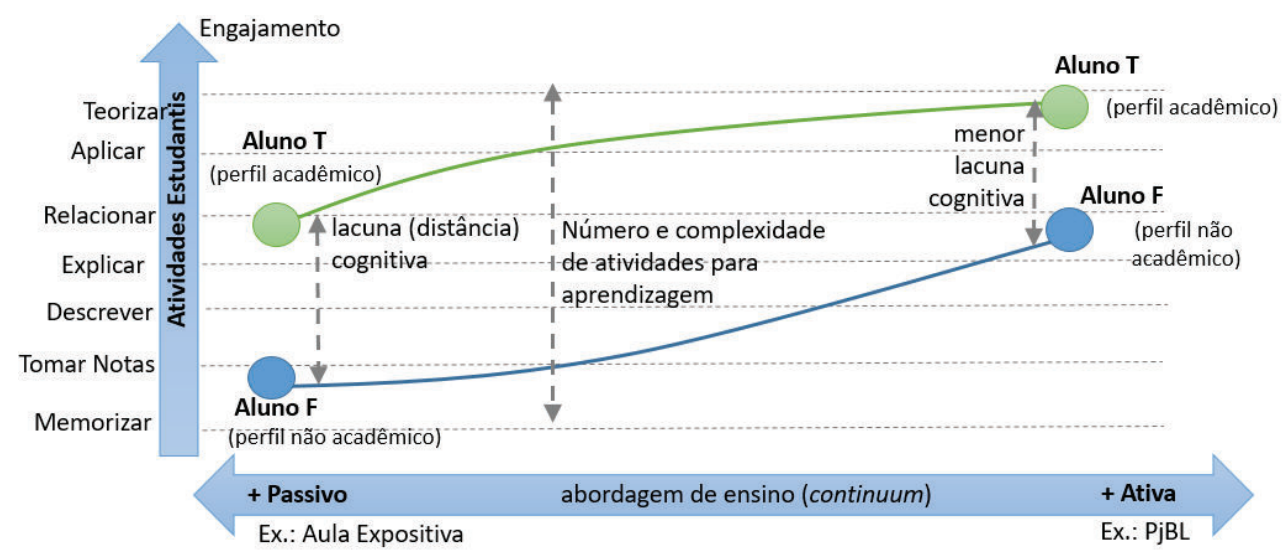

Figura 1. Continuum educacional aplicado à educação em programação

Na figura 1 nota-se uma relação direta entre os métodos de ensinos adotados, no eixo $\mathrm{X}$, e respectivos fenômenos, visualizados no eixo Y. Assim, ao tornar a experiência educacional mais construtivista por meio de PjBL há uma sinergia e vários aspectos dos alunos melhoram: aumenta a capacidade de realização de atividades educacionais, aumenta o engajamento e diminui a lacuna cognitiva. Os benefícios tendem a retirar o perfil $\mathrm{F}$ da região de notas de reprovação. Assim, diante das modificações de métodos no continuum educacional de programação de computadores, o que avaliar, e como avaliar, na experiência educacional tradicional versus PjBL ? 


\section{O ensaio}

Nesta seção, são apresentadas as propostas para o que avaliar e como avaliar relativas ao EA-PROG. Iniciando pela aprendizagem, a tabela 1 resume a proposta das avaliações auxiliares e alguns exemplos ilustrativos. Sugere-se ainda que as avaliações auxiliares sejam divididas em três categorias: do indivíduo, dos grupos e do coletivo. Além disso, propõe-se que tais avaliações auxiliares sejam classificadas em dois tipos. Primeiro tipo, avaliações psicométricas, tais como motivação e engajamento. Segundo tipo, avaliações de desempenho do coletivo, a exemplo da lacuna cognitiva em Biggs e Tang (2011).

Tabela 1. Proposta para avaliação da aprendizagem em programação de computadores

\begin{tabular}{|c|c|c|c|}
\hline Abordagem & Medida Individual & Medida de Grupo & Medida do Coletivo \\
\hline Tradicional & $\begin{array}{l}\text { O que: Nota, atividades } \\
\text { estudantis. } \\
\text { Como: Prova, teste, } \\
\text { relatório, apresentação ou } \\
\text { trabalho individual. }\end{array}$ & $\begin{array}{l}\text { O que: Nota, atividades } \\
\text { estudantis. } \\
\text { Como: Trabalho, } \\
\text { apresentação, seminário, } \\
\text { provas em grupo. }\end{array}$ & Não encontrado. \\
\hline $\mathrm{PjBL}$ & $\begin{array}{l}\text { Avaliações auxiliares: } \\
\text { avaliações psicométricas } \\
\text { individuais, tais como } \\
\text { motivação, engajamento, } \\
\text { comunicação, capacidade } \\
\text { de planejamento, de } \\
\text { execução, de contribuição } \\
\text { ao coletivo. } \\
\text { Adicionalmente, lacuna } \\
\text { cognitiva do indivíduo para } \\
\text { com o grupo. } \\
\text { Como: Construtos e/ou } \\
\text { projetos. }\end{array}$ & $\begin{array}{l}\text { Avaliações auxiliares: } \\
\text { psicométricas coletivas, tais } \\
\text { como capacidade de } \\
\text { trabalho em equipe, de } \\
\text { convencimento. } \\
\text { Adicionalmente, lacuna } \\
\text { cognitiva entre grupos. } \\
\text { Como: Construtos e/ou } \\
\text { projetos. Ex.: subprojetos } \\
\text { interdependentes, rotação de } \\
\text { projetos entre grupos. }\end{array}$ & $\begin{array}{l}\text { Avaliações auxiliares: } \\
\text { motivação, engajamento, } \\
\text { entre outros aspectos } \\
\text { psicométricos. Lacuna } \\
\text { cognitiva do coletivo } \\
\text { discente. } \\
\text { Como: Construtos e/ou } \\
\text { projetos. Ex.: projeto da } \\
\text { sala como grupo único, } \\
\text { projeto do curso, projetos } \\
\text { de turmas anteriores, } \\
\text { contribuições de sujeitos } \\
\text { externos à sala de aula. } \\
\end{array}$ \\
\hline
\end{tabular}

$\mathrm{Na}$ tabela 1, para avaliações psicométricas, existem diversos instrumentos psicométricos, ou construtos. Assim, é sugerido classificar estes construtos com relação às necessidades da pesquisa em dois diferentes tipos: construtos específicos ou construtos abrangentes. Como exemplo de construto específico, há o de avaliação de motivação em programação, disponível na pesquisa de Santana, Figuerêdo e Bittercour (2017). Outro exemplo é o Ticket Driven Development (desenvolvimento orientado a bilhete, tradução nossa), que avalia a contribuição do indivíduo para com o grupo de estudo por meio de bilhetes que descrevem as atividades esperadas de cada aluno [Igaki et al. 2014]. Segundo, como exemplo de construto abrangente, há um instrumento para validação da motivação no ensino superior, que mensura a motivação extrínseca e intrínseca dos alunos [Boruchovitch 2008]. Existem, assim, diversos instrumentos destinados à coleta de avaliações auxiliares. Estes podem inclusive vir das áreas humanas, tais como a Psicologia e, ainda assim, servirem para avaliação da aprendizagem por meio de $\mathrm{PjBL}$. Portanto, sempre que possível, é sugerida a opção por construtos reutilizáveis de outras pesquisas, de modo a simplificar, acelerar e padronizar futuras pesquisas, em detrimento da criação de novos construtos.

Relativo à lacuna cognitiva, em Biggs e Tang (2011) não há uma avaliação definida para como medi-la. Assim, propõe-se que a lacuna cognitiva no EA-PROG pode ser avaliada estatisticamente pela distância geométrica entre dois centros de gravidades: dos centros dos perfis F e T. A título de exemplo ilustrativo, dadas as notas dos alunos, e 
assumindo que é possível classificar os alunos em perfis $\mathrm{T}$ e $\mathrm{F}$, uma lacuna cognitiva de valor igual a zero significa que os rendimentos escolares foram similares (notas estatisticamente iguais). A medida que a distância geométrica das notas destes perfis $\mathrm{T}$ e F aumenta, a lacuna cognitiva assume valor diferente de zero. Este valor representaria a lacuna cognitiva ilustrada na Figura 1 e referenciada na Tabela 1.

Propõe-se também que os projetos, por si só, podem se configurar instrumentos de avaliações auxiliares, conforme Tabela 1. Quando os docentes de um estudo descrevem a motivação e alegria dos estudantes ao verem seu projeto $\mathrm{PjBL}$ realizado com sucesso [Kastl et al. 2016], há neste relato uma clara avaliação auxiliar, mesmo que qualitativa. Outro relato similar foi a inclusão da satisfação de um cliente externo à aula como parte da composição da nota final. Outro exemplo é o envolvimento dos demais sujeitos do processo educacional, tais como alunos, grupos e pessoas externas às aulas, todos contribuindo para a composição das notas. Ou relatos da autoavaliação do aluno visando compor avaliações auxiliares ou sua própria nota [Abdool and Pooransingh 2014; Francese et al. 2015]. Todas estas avaliações auxiliares podem ter diversos ângulos, podem ser agregadas ou não às notas. Em $\mathrm{PjBL}$, os projetos são a espinha dorsal pelo qual o EA-PROG acontece. No entanto, sempre se deve observar a aprendizagem como objetivo final da experiência educacional.

No que se refere à medição do ensino, existem diversas dificuldades e aspectos a serem considerados nas aferições de intervenções metodológicas de ensino [Bordenave and Pereira 1991]. Uma alta complexidade nas inovações ou nas avaliações educacionais pode desestimular ou até mesmo inviabilizar pesquisas na educação [Bell 2016]. Assim, é proposta uma avaliação simplificada nomeada experiência educacional. Esta é uma resultante em escala Likert do quão construtivista ou quão instrutivista foi a experiência dos alunos com base no tempo de aula. Mais especificamente, a medição da experiência educacional ser aferida por uma escala de cinco pontos, dentro do continuum (ou gradiente) educacional. Este gradiente é classificado em escala ordinal por meio de cinco letras, nesta sequência: I, i, ic, c e C. A letra (I), significa totalmente instrutivista, equivale a menos dois pontos e representa o polo instrutivista. Menos um ou sigla (i), representa um método predominantemente instrutivista. A sigla (ic) representa uma experiência educacional próxima ao centro do gradiente e, portanto, igualmente instrutivista e construtivista. E assim por diante, até chegar ao outro lado do gradiente, com a letra (C), que significa uma experiência próxima ao polo construtivista. Valores intermediários devem ser arredondados por meio da regra matemática padrão.

Também se propõe uma avaliação ordinal a ser agregada à experiência educacional: recursos educacionais PjBL para o EA-PROG. Estes recursos podem ser de dois tipos: físicos ou digitais. Visam mensurar o quão preparados estão os ambientes físico e digital à implantação de PjBL. Exemplos destes recursos podem ser: presença de salas de aula não lineares ou salas de reunião coletiva; acesso a espaços exteriores à sala de aula, tais como a comunidade ou empresas; ferramentas digitais de gestão de projetos.

A tabela 2 sintetiza a proposta do que avaliar, e de como avaliar, relativos aos dois aspectos ensino e aprendizagem de programação de computadores. Primeiro, relativo ao ensino é proposto avaliar o grau de experiência educacional e respectivos recursos educacionais. Segundo, relativo à aprendizagem, é proposto a utilização de avaliações auxiliares, em complemento as avaliações tradicionais requeridas por Lei: 
Tabela 2. Resumo das variáveis para medição do EA-PROG por meio de PjBL

\begin{tabular}{|c|c|c|c|c|}
\hline EA-PROG & Nome & Tipo & Níveis e intervalos & Como (método) \\
\hline Ensino & $\begin{array}{l}\text { Recursos } \\
\text { Educacionais } \\
\text { para PjBL }\end{array}$ & Ordinal & $\begin{array}{l}\text { (0) ausente; } \\
\text { (1) parcial; } \\
\text { (2) presente. }\end{array}$ & $\begin{array}{l}\text { Aferição da presença de recursos } \\
\text { para EA-PROG com PjBL nos } \\
\text { ambientes físicos e digitais. }\end{array}$ \\
\hline Ensino & $\begin{array}{l}\text { Experiência } \\
\text { Educacional } \\
\text { em PjBL }\end{array}$ & Ordinal & $\begin{array}{l}\text { (I) muito } \\
\text { instrutivista; } \\
\text { (i) moderadamente } \\
\text { instrutivista; } \\
\text { (ic) igualmente } \\
\text { instrutivista e } \\
\text { construtivista; } \\
\text { (c) moderadamente } \\
\text { construtivista; } \\
\text { (C) muito } \\
\text { construtivista. }\end{array}$ & $\begin{array}{l}\text { Escala Likert de cinco pontos, } \\
\text { variando de menos dois a mais dois. } \\
\text { Avalia os métodos educacionais } \\
\text { com base no tempo de aula. Por } \\
\text { exemplo, dadas quatro aulas } \\
\text { possíveis, se três foram atividades } \\
\text { coletivas por meio de PjBL e se } \\
\text { uma foi expositiva então a } \\
\text { resultante é uma experiência } \\
\text { educacional "c" (moderadamente } \\
\text { construtivista), ou seja, } 75 \% \text { do } \\
\text { tempo de atividades construtivistas. }\end{array}$ \\
\hline Aprendizagem & $\begin{array}{l}\text { Medida } \\
\text { Auxiliar: } \\
\text { Psicométrica }\end{array}$ & Racional & 0 a 100 & $\begin{array}{l}\text { Utilização de construtos } \\
\text { psicométricos, preferencialmente } \\
\text { validados no contexto nacional, tais } \\
\text { como Motivação [Boruchovitch } \\
\text { 2008] ou engajamento [Stelko- } \\
\text { Pereira et al. 2015]. }\end{array}$ \\
\hline Aprendizagem & $\begin{array}{l}\text { Medida } \\
\text { Auxiliar: } \\
\text { Desempenho } \\
\text { do Coletivo }\end{array}$ & Racional & 0 a 100 & $\begin{array}{l}\text { Lacuna Cognitiva por meio da } \\
\text { distância geométrica entre grupos ou } \\
\text { indivíduos. Quanto menor o valor, } \\
\text { menor a lacuna cognitiva. }\end{array}$ \\
\hline
\end{tabular}

\section{Limitações e Discussões}

Existem limitações ao escopo deste ensaio. Uma relevante limitação está relacionado ao fato de que o método de ensino e os métodos de avaliação da aprendizagem não são os únicos fatores de impacto no ensino de graduação [Bordenave and Pereira 1991; Queirós 2014]. Adicionalmente, as causas sociais não atuam de modo isolado, como é o caso da educação. Além disso, há de se considerar limitações e cuidados ao se estabelecer casualidade e implicações na área educacional [Bell 2016]. Estes fatos impõem limites à amplitude e à profundidade das proposições, configurando tópicos para estudos futuros.

Há também diversas discussões a respeito do tema. Estas podem ser tomadas desde a perspectiva do indivíduo até à perspectiva da soberania nacional. Relativo aos estudantes, depois de vários anos utilizando a abordagem tradicional de EA-PROG, chegam ao mercado com uma alta bagagem teórica, mas lacunas em áreas-chave do EAPROG. Estão familiarizados com conceitos abstratos e de difícil compreensão, porém não são raras as queixas de empresas a respeito do pouco conhecimento prático dos alunos [Zhang and Liu 2012]. São relatadas dificuldades não técnicas destes alunos, tais como a baixa capacidade de comunicação, de trabalho em equipe ou de resolução de problemas [Morimoto 2016]. Talvez por isso avaliações auxiliares tentem entender este fenômeno em sala de aula, sendo um relevante tópico para estudos futuros.

Se estas lacunas dificultam o acesso dos alunos ao mercado de trabalho no contexto em que foram estudados [Morimoto 2016; Zhang and Liu 2012], de modo análogo, no contexto da soberania nacional, há relatos da ligação entre $\mathrm{o}$ 
subdesenvolvimento econômico versus a capacidade de inovação educacional da Nação. Novos métodos de ensino e aprendizagem são fatores-chave para o progresso de uma nação [Porcaro 2011]. Assim, podem ser tópicos de interesse a utilização de PjBL no EAPROG como meio para: a redução de lacunas de empregabilidade; a orientação e o fomento de programas educacionais para necessidades regionais; uma maior integração com mercados locais ou internacionais de trabalho.

Por fim, são igualmente relevantes as limitações para as avaliações utilizadas no contexto PjBL. No ensino tradicional, usualmente avalia-se o resultado das ações de um aluno [Luckesi 2008] tais como resultados de provas, trabalhos ou projetos. Pesquisas futuras poderiam investigar os métodos, pesos, ponderações e instrumentos mais adequados ao EA-PROG por meio de PjBL. As avaliações também poderiam ser adaptadas ou ponderadas de acordo com o grau da experiência educacional. Ou ainda, baseado em diferentes perfis de alunos, investigar quais avaliações e adaptações podem ocorrer para o acolhimento das diferenças como fator de sinergia educacional, trazendo à rotina acadêmica a pluralidade usualmente encontrada em projetos do mundo real.

\section{Conclusão}

São vários os relatos e pesquisas a respeito do tema de inovação educacional por meio de PjBL para a programação de computadores, com relevante dedicação ao fenômeno de benefícios aos alunos. Estes benefícios estão relacionados às avaliações tradicionais, na forma de notas ou presença dos alunos. Ou relacionados às avaliações auxiliares: i) melhorar aspectos psicométricos - a motivação, a capacidade de trabalho em equipe, a comunicação, entre outros; ou ii) reduzir a lacuna cognitiva dos alunos, a exemplo do fenômeno de redução da variância no rendimento escolar.

Neste desafiante contexto, este ensaio analisou a padronização sobre o que avaliar, e como avaliar, no EA-PROG por meio de PjBL. Com relação ao ensino, foi proposto avaliar os métodos de ensino e seus recursos educacionais. Com relação à aprendizagem, foram propostas avaliações auxiliares. Estas podem ser agrupadas em: avaliações psicométricas, como a motivação; ou avaliações do desempenho coletivo, a exemplo da lacuna cognitiva. Podem também ser classificadas em três tipos: individuais, dos grupos e do coletivo. Foram abordados exemplos de avaliações auxiliares, sem, no entanto, exaurir a discussão. Adicionalmente, foram feitas propostas para futuras pesquisas.

Uma eventual padronização destas avaliações poderia colaborar com a simplificação da coleta, da análise e do compartilhamento de dados nesta linha de pesquisa. A padronização também poderia contribuir para criação de ferramentas digitais reaproveitáveis, ou melhorar a integração da programação de computadores com outras áreas interdisciplinares. Em suma, esta padronização poderia colaborar com a identificação de benefícios adicionais aos alunos, ajudar no combate à repetência e à evasão, contribuindo para uma melhor formação de futuros programadores.

\section{Referências Bibliográficas}

Abdool, A. and Pooransingh, A. (2014). An Industry-Mentored Undergraduate Software Engineering Project. In 2014 IEEE Frontiers in Education Conference (FIE). , Frontiers in Education Conference. IEEE.

Beck, C. and Kosnik, C. (2012). Innovations in Teacher Education: A Social Constructivist Approach. SUNY series, Teacher Preparation and Development. New 
York: State University of New York Press. p. 7-23.

Bell, J. (2016). Projeto de Pesquisa: guia para pesquisadores iniciantes em educação, saúde e ciências sociais. 4. ed. São Paulo: Artmed Editora. p. 21-22.

Biggs, J. B. and Tang, C. S. (2011). Teaching for quality learning at university. SRHE and Open University Press imprint. SRHE and Open University Press Imprint. 4. ed. New York, USA: McGraw-Hill Education. p. 1-16.

Bordenave, J. D. and Pereira, A. M. (1991). Estratégias de ensino-aprendizagem. 12. ed. Petrópolis: Vozes.

Boruchovitch, E. (2008). Escala de Motivação Para Aprender de Universitários: Propriedades Psicométricas. Avaliação psicológica, v. 7, n. 2, p. 127-134.

BRASIL (1996). Lei $\mathrm{n}^{0}$ 9.394, de 20 de dezembro de 1996. Brasil. http://www.planalto.gov.br/ccivil_03/leis/L9394.htm.

Chan Mow, I. T. (2008). Issues and difficulties in teaching novice computer programming. In: Iskander, M.[Ed.]. . Innovative Techniques in Instruction Technology, E-Learning, E-Assessment, and Education. Dordrecht: Springer Netherlands. p. 199-204.

Corno, F. and De Russis, L. (2017). Training Engineers for the Ambient Intelligence Challenge. IEEE Transactions on Education, v. 60, n. 1, SI, p. 40-49.

DeFillippi, R. J. (2001). Introduction: Project-Based Learning, Reflective Practices and Learning. Management Learning, v. 32, n. 1, p. 5-10.

Fior, C. A. and Mercuri, E. (2013). Evidências de validade da Escala de Envolvimento Acadêmico para universitários. Avaliação Psicológica, v. 12, n. 1, p. 81-89.

Francese, R., Gravino, C., Risi, M., Scanniello, G. and Tortora, G. (2015). Using ProjectBased-Learning in a mobile application development course-An experience report. Journal of Visual Languages \& Computing, v. 31, p. 196-205.

Giraffa, L. M. M., Moraes, M. C. and Uden, L. (2014). Teaching Object-Oriented Programming in First-Year Undergraduate Courses Supported By Virtual Classrooms. In: Uden, L.; Tao, Y.-H.; Yang, H.-C.; Ting, I.-H.[Eds.]. . The 2nd International Workshop on Learning Technology for Education in Cloud. Dordrecht: Springer Netherlands. p. 15-26.

Goulding, T. (2013). A first semester freshman project: The enigma encryption system in C. ACM Inroads, v. 4, n. 1, p. 43-46.

Greening, T. (2012). Computer Science Education in the 21st Century. In: Springer Science \& Business Media[Ed.]. . Ballarat: Springer New York. p. 47-59.

Guzman-Ramirez, E. and Garcia, I. A. (2013). Using the Project-Based Learning Approach for Incorporating an FPGA-Based Integrated Hardware/Software Tool for Implementing and Evaluating Image Processing Algorithms Into Graduate Level Courses. Computer Applications in Engineering Education, v. 21, n. 1, p. E73-E88.

Igaki, H., Fukuyasu, N., Saiki, S., Matsumoto, S. and Kusumoto, S. (2014). Quantitative Assessment with Using Ticket Driven Development for Teaching Scrum Framework. [A. Jalote, P and Briand, L and VanDerHoek, Ed.]In 36th International Conference on Software Engineering (ICSE Companion 2014). . Association for Computing 
Machinery.

Iskander, M. (2008). Innovative Techniques in Instruction Technology, E-learning, Eassessment and Education. SpringerLink: Springer e-Books. New York: Springer Netherlands. p. 199-203.

Kastl, P., Kiesmüller, U. and Romeike, R. (2016). Starting out with projects - Experiences with agile software development in high schools. [B. E. Vahrenhold J. Barendsen E., Ed.]In ACM International Conference Proceeding Series. . Association for Computing Machinery.

Luckesi, C. C. (2008). Avaliação da Aprendizagem Escolar: estudos e proposições. Cortez Editora.

Manogaran, E. (2013). ACt-PBL: An adaptive approach to teach multi-core computing in university education. In Proceedings - 2013 IEEE 5th International Conference on Technology for Education, T4E 2013. . IEEE Computer Society.

Mioduser, D. and Betzer, N. (2007). The contribution of Project-based-learning to highachievers' acquisition of technological knowledge and skills. Int J Technol Des Educ, v. 77, n. 27.

Morimoto, C. (2016). Improvement of IT Students' Communication Skills using Project Based Learning. In Proceedings of the 8th International Conference on Computer Supported Education. . SciTePress.

Payne, C. R. (2009). Information Technology and Constructivism in Higher Education: Progressive Learning Frameworks. Hershey, PA, USA: IGI Global. p. 1-25.

Porcaro, D. (2011). Applying constructivism in instructivist learning cultures. Multicultural Education \& Technology Journal, v. 5, n. 1, p. 39-54.

Queirós, R. (2014). Innovative Teaching Strategies and New Learning Paradigms in Computer Programming. Advances in higher education and professional development (AHEPD) book series. Hershey, PA, USA: IGI Global. p. 131-133.

Romeike, R. and Göttel, T. (2012). Agile projects in high school computing education Emphasizing a learners' perspective. In ACM International Conference Proceeding Series.

Santana, Bianca L; Figuerêdo, José S; Bittencour, R. A. (2017). Motivação de Estudantes Non-Majors em uma Disciplina de Programação. $25^{\circ}$ WEI - Workshop sobre Educação em Computação, p. 2287-2296.

Silva Filho, R. L. L. e, Motejunas, P. R., Hipólito, O. and Lobo, M. B. D. C. M. (2007). A evasão no ensino superior brasileiro. Cadernos de Pesquisa, v. 37, n. 132, p. 641659.

Stelko-Pereira, A. C., Valle, J. E. and Williams, L. C. A. (2015). Escala de engajamento escolar: análise de características psicométricas. Avaliação Psicológica, v. 14, n. 2, p. 207-212.

Zhang, Y. and Liu, Y. (2012). Management enhanced double PBL based reform in advanced programming design course. In Proceedings of the 14th IEEE International Conference on High Performance Computing and Communications, HPCC-2012 - 9th IEEE International Conference on Embedded Software and Systems, ICESS-2012. 